\title{
Risk/benefit tradeoff of habitual physical activity and air pollution on chronic pulmonary obstructive disease: findings from a large prospective cohort study
}

\author{
Lan Chen ${ }^{1}$, Miao Cai ${ }^{1}$, Haitao Li ${ }^{2}$, Xiaojie Wang ${ }^{1}$, Fei Tian ${ }^{1}$, Yinglin Wu${ }^{1}$, Zilong Zhang ${ }^{1 *}$ and Hualiang Lin ${ }^{1 *}$
}

\begin{abstract}
Background: The combined health impact of physical activity (PA) and air pollution on chronic obstructive pulmonary disease (COPD) remains unclear. We investigated the joint effects of habitual PA and long-term fine particulate matter $\left(\mathrm{PM}_{2.5}\right)$ exposure on COPD incidence in a prospective population-based cohort.
\end{abstract}

Methods: A prospective cohort study was conducted using data from the UK Biobank. Incidence of COPD was ascertained through linkage to the UK National Health Services register. Annual mean PM ${ }_{25}$ concentration was obtained using land use regression model. PA was measured by questionnaire and wrist-worn accelerometer. Cox proportional hazard models were applied to examine the associations between $\mathrm{PM}_{2.5}, \mathrm{PA}$, and COPD. Additive and multiplicative interactions were examined.

Results: A total of 266,280 participants free of COPD at baseline were included in data analysis with an average follow-up of 10.64 years, contributing to around 2.8 million person-years. Compared with participants with low level of PA, those with higher PA levels had lower risks of COPD incidence [hazard ratio (HR): 0.769, 95\% Cl: 0.720, 0.820 for moderate level; HR: $0.726,95 \%$ Cl: $0.679,0.776$ for high level]. By contrast, $\mathrm{PM}_{2.5}$ was associated with increased risk of COPD (HR per interquartile range increment: 1.065, 95\% Cl: 1.032, 1.099). Limited evidence of interaction between habitual PA and $\mathrm{PM}_{25}$ exposure was found. Similar results were found for accelerometer-measured PA.

Conclusions: Our study suggests that habitual PA could reduce risk of COPD incidence, and such protective effects were not affected by ambient $\mathrm{PM}_{25}$ pollution exposure.

Keywords: Air pollution, Physical activity, Chronic obstructive pulmonary disease, Cohort study

\section{Background}

Chronic obstructive pulmonary disease (COPD) is a leading cause of global disease burden [1]. According to the Global Burden of Diseases Study (GBD) 2017, COPD remained the most prevalent chronic respiratory

\footnotetext{
*Correspondence: zhangzilong@mail.sysu.edu.cn; linhualiang@mail.sysu.edu. $\mathrm{cn}$

${ }^{1}$ Department of Epidemiology, School of Public Health, Sun Yat-sen University, Guangzhou 510080, China

Full list of author information is available at the end of the article
}

disease worldwide, accounting for over $50 \%$ of chronic respiratory disease cases globally [2]. Besides, COPD has become the fifth leading cause of disability-adjusted lifeyears (DALYs) worldwide, responsible for approximately 81.6 million DALYs in 2017 [3].

A number of risk factors concerning the occurrence of COPD have been well documented, including genetics, smoking, occupational exposure, chronic asthma, and so on [4]. An increasing body of studies have also indicated that air pollution is an important 


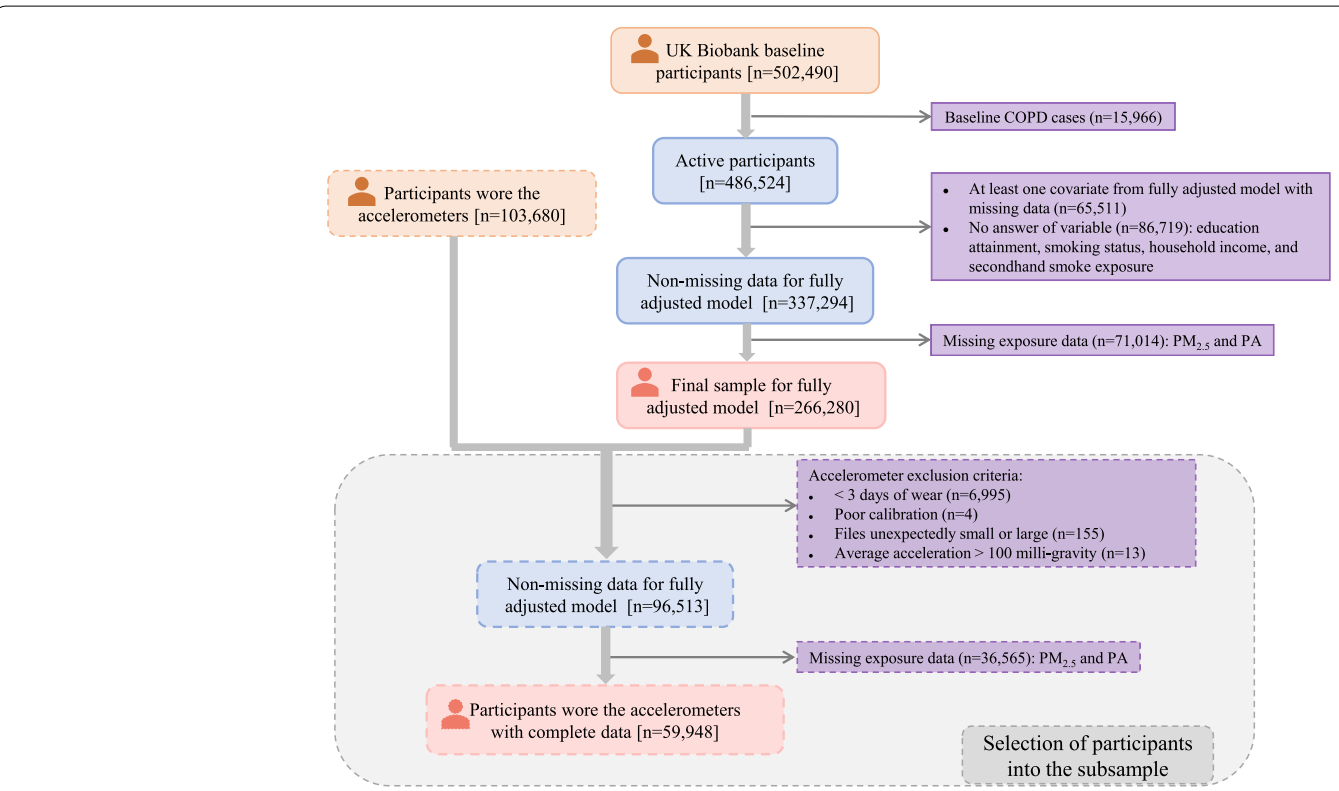

Fig. 1 Selection of participants. Abbreviations: COPD, chronic obstructive pulmonary disease; $\mathrm{PM}_{2.5}$, particulate matter with an aerodynamic diameter smaller than $2.5 \mu \mathrm{m}$; PA, physical activity

risk factor of COPD, especially particulate matter with an aerodynamic diameter smaller than 2.5 $\mu \mathrm{m}\left(\mathrm{PM}_{2.5}\right)$ [5-7]. By contrast, physical activity (PA) has been shown as an effective measure to prevent non-communicable diseases, including COPD [8]. Exercising may increase the inhalation of air pollutants because of higher ventilation, and consequently amplify the adverse health effects of air pollutants [9]. Therefore, balancing the hazard/benefit tradeoff of air pollution and PA has become an important public issue since over $90 \%$ of the population worldwide lives in countries where air quality fails to meet the World Health Organization (WHO) guidelines [10]. Apart from inconsistency in current findings on the combined health impact of PA and air pollution [11], most previous studies solely depended on self-reported PA, which is subject to limitations of reliability and validity [12]. Recent technological advances have made wearable motion sensors, such as accelerometers, portable and convenient to use in large epidemiological studies, allowing more accurate measurement of PA with different intensities [13].

In the present study, we investigated the combined health impact of habitual PA (measured by both questionnaire and accelerometer) and chronic exposure to $\mathrm{PM}_{2.5}$ on COPD incidence in a prospective cohort, in an effort to bring out more solid clues for the health effects of PA on COPD with consideration of air pollution.

\section{Methods}

\section{Study population}

Our study applied data from the UK Biobank Cohort study [Application Number: 69550], an ongoing longitudinal cohort of over 0.5 million participants aged 40-69 years at baseline (2006-2010). The study was mainly conducted in urban areas of England, Scotland, and Wales through the UK National Health Services register [6]. All participants completed a series of baseline assessment, including socioeconomic factors, lifestyle and behavioral factors, and the history of medication and operations. Biological samples, such as blood and urine samples, were taken as well. Details of the UK Biobank protocols can be found elsewhere [14]. The UK Biobank study was approved by the North West Multi-centre Research Ethics Committee (06/MRE08/65). Informed consent was obtained from all participants [15].

Inclusion and exclusion procedures of the study population in the present study are outlined in Fig. 1. Of the 502,490 participants in the UK Biobank, 15,966 with prevalent COPD at baseline were excluded. We further excluded 149,230 participants due to missing data for at least one covariate in fully adjusted models, leaving 337,294 participants with complete covariate data. After further excluding 71,014 participants with missing $\mathrm{PM}_{2.5}$ or PA, 266,280 participants were left in our final sample. As for participants with objectively measured PA, according to data exclusion criteria of accelerometers in previous studies $[16,17]$ and following the same selection 
procedures as participants with self-reported PA, a total of 59,948 participants were left in the subsample analysis.

\section{Measurement of habitual physical activity (PA)}

Information on habitual PA was obtained by a selfadministered questionnaire and wrist-worn accelerometer. Briefly, self-reported PA was ascertained with a modified version of the International Physical Activity Questionnaire (IPAQ), which includes six questions about duration and frequency of walking, moderateintensity and vigorous-intensity exercise undergone in the last 4 weeks [18]. Each intensity was assigned a corresponding metabolic equivalent (MET, $1 \mathrm{MET}=1 \mathrm{kil}-$ ocalorie per hour per kilogram of bodyweight): 3.3 for walking, 4.0 for moderate PA, and 8.0 for vigorous PA [19]. We then quantified PA of each participant by calculating minutes of MET each week (MET-min/week) based on the reported intensity, duration, and frequency of PA in 1 week. Afterwards, participants were classified into three groups based on a standard scoring criteria of International Physical Activity Questionnaire (IPAQ) [20]: low (< 600 MET-min/week), moderate (600 to 3000 MET-min/week), and high ( $\geq 3000$ MET-min/week). The threshold of $600 \mathrm{MET}-\mathrm{min} /$ week is equal to reaching the recommended $\mathrm{WHO}$ guidelines for moderate-intensity PA (150 min per week) [21].

Furthermore, we adopted a subsample of about 100,000 participants with objectively measured PA using triaxial accelerometers (Axivity AX3, Newcastle upon Tyne, UK). Participants were chosen voluntarily and accelerometers (the Axivity AX3 wrist-worn triaxial accelerometer) were sent to their personal addresses [22]. They were required to wear accelerometers on their dominant wrists for seven consecutive days. Information on the accelerometer protocol, including data extraction and processing, has been documented in detail elsewhere [23]. Individuals were grouped into three groups based on the PA tertiles [low $<24.32$ milli-gravity $(\mathrm{mg})$, moderate 24.32 to $30.70 \mathrm{mg}$, and high $\geq 30.70 \mathrm{mg}$ ] [24].

\section{Air pollution assessment}

The annual average concentration of $\mathrm{PM}_{2.5}$ in 2010 was calculated using a land-use regression model (LUR) developed by the European Study of Cohorts for Air Pollution Effects (ESCAPE) project [25]. Details on the development and validation of the ESCAPE LUR models have been described elsewhere [26]. In brief, based on a range of predictive variables (such as traffic intensity, population, topography, and land use) derived from geographic information system (GIS), LUR models were used to calculate the spatial variation in air pollutant concentrations at individuals' residential addresses provided at baseline. Leave-one-out cross-validation showed good model performance for $\mathrm{PM}_{2.5}$ (cross-validation $R^{2}=77 \%$ ) in the southeast England area (London/ Oxford) [27]. The obtained $\mathrm{PM}_{2.5}$ concentration in 2010 was used as a surrogate measure of long-term $\mathrm{PM}_{2.5}$ exposure, given the fluctuation of temporal trend of $\mathrm{PM}_{2.5}$ concentrations remained generally parallel during study period $[28,29]$.

\section{Outcome ascertainment}

Participants were followed up from enrollment till the first occurrence of COPD, death, loss of follow-up, or 31 December 2019, whichever came first. COPD cases were identified using self-reported information, primary care data, and hospital admission data through linkage to the UK National Health Services register [18]. COPD diagnoses were coded as J40-J44 according to the International Classification of Diseases version-10 (ICD-10).

\section{Covariates}

Potential confounders were selected based on literature review a priori $[6,11,30]$, including demographic characteristics (sex, age, ethnicity, etc.), lifestyle factors (smoking status, drinking status, and fruit and vegetable intake), and so on. Details of definitions of these covariates could be seen in the Additional file 1: Supplementary methods of covariates and 4-model analytical protocol [14, 21, 31-33].

\section{Statistical analysis}

Cox proportional hazard regression models with followup time as time scale were applied to examine the associations of long-term $\mathrm{PM}_{2.5}$ exposure and habitual PA with COPD incidence. STROBE cohort reporting guidelines were adopted as well [34]. We developed a 4-model analytical protocol by adding covariates (e.g., demographic characteristics, socioeconomic factors, lifestyle factors) incrementally. We investigated the independent associations of PA and $\mathrm{PM}_{2.5}$ first by building Models 1, 2, and 3 . Then Model 4 was constructed for mutual adjustment. Details of the 4-model analytical protocol can be found in Additional file 1: Supplementary methods of covariates and 4-model analytical protocol.

When exposures were included as continuous variables, hazard ratio, and 95\% confidence interval (HR, 95\% $\mathrm{CI}$ ) were calculated for each inter-quantile range (IQR) increment in $\mathrm{PM}_{2.5}\left(1.27 \mu \mathrm{g} / \mathrm{m}^{3}\right)$ and per $600 \mathrm{MET}-\mathrm{min} /$ week in PA. For categorical exposures, effect estimates were calculated with reference to low level of PA or the first tertile of $\mathrm{PM}_{2.5}$. The proportional hazard assumption was examined by plotting Schoenfeld residuals, and no evidence of serious violation was found. Concentrationresponse relationship between long-term $\mathrm{PM}_{2.5}$ exposure and COPD incidence was examined using a spline term 
in Cox model, where $\mathrm{PM}_{2.5}$ was treated as a continuous variable with a degree of freedom (df) of 4 .

Subgroup analyses stratified by $\mathrm{PM}_{2.5}$ tertiles $(<9.48 \mu \mathrm{g} /$ $\mathrm{m}^{3}, 9.48$ to $10.27 \mu \mathrm{g} / \mathrm{m}^{3}$, and $\geq 10.27 \mu \mathrm{g} / \mathrm{m}^{3}$ ) or PA levels (low, moderate, and high) were also conducted using Model 3. Cochran-Armitage trend test was used to confirm the constant trend toward higher incidence of COPD with an increasing PA levels and $\mathrm{PM}_{2.5}$ exposure levels [35].

We further investigated the potential interactions between $\mathrm{PA}$ and $\mathrm{PM}_{2.5}$ exposure on both additive and multiplicative scales. For additive interaction, we firstly categorized study participants into three groups based on their $\mathrm{PM}_{2.5}$ tertiles (low $<9.48 \mu \mathrm{g} / \mathrm{m}^{3}$, moderate 9.48 to $10.27 \mu \mathrm{g} / \mathrm{m}^{3}$, and high $\geq 10.27 \mu \mathrm{g} / \mathrm{m}^{3}$ ). Combined with PA levels (low, moderate, and high), we then created a new term with nine categories representing nine combinations $(3 \times 3)$ of $\mathrm{PM}_{2.5}$ exposure levels and PA levels.

To test the additive interaction, we calculated the relative excess risk due to interaction (RERI) and their corresponding confidence intervals $(\mathrm{CI})$ with the reference group of high level of $\mathrm{PM}_{2.5}$ exposure and low level of PA. RERI measures the combined excess risk in both exposed group that is due to the interaction [36]. A RERI of 0 denotes no additive interaction (i.e., the combined excess risk is the sum of their individual excess risks, and the excess risk is calculated as HR-1), a RERI of more than 0 indicates positive interaction (i.e., the combined excess risk is more than the sum of their individual excess risks), and a RERI of less than 0 implies that the combined excess risk is less than the sum of their individual excess risks [37]. For example, a negative RERI value in the present study would indicate that the benefit of PA was attenuated by residential $\mathrm{PM}_{2.5}$ exposure.

On multiplicative scale, we added a product term between $\mathrm{PM}_{2.5}$ exposure and PA levels. Likelihood tests were applied to test the significance of interaction term by comparing the model with and without the interaction term. A $p$ value of the interaction term less than 0.05 indicates a multiplicative interaction [38].

For objectively measured PA data extracted from Axivity AX3, we used average acceleration to represent the overall PA intensity and categorized participants into three levels [ $<24.32$ milli-gravity (mg), 24.32 to $30.70 \mathrm{mg}$, and $\geq 30.70 \mathrm{mg}$ ]. Detailed information of accelerometermeasured PA level can be found elsewhere [24, 39]. The analyzing protocol for objectively measured PA was the same with that of the self-reported PA.

\section{Sensitivity analysis}

We performed a number of sensitivity analyses to examine the robustness of our results. First, we additionally adjusted for baseline lung function, inverse distance to main road, and both [40, 41]. These two variables were weakly correlated with $\mathrm{PM}_{2.5}$ and PA (Additional file 1: Table S1). Second, instead of treating death as censored in our main analysis, we treated it as a competing risk and conducted an additional sensitivity analysis using Fine-Gray subdistribution hazards regression model [42]. Third, given the exclusion of a large number of participants with missing information, missing values of the baseline covariates were imputed with multivariate imputation via chained equation (MICE) (the number of imputations was 5) to make sure that the representativeness of the cohort was not substantially affected by exclusion of participants [18].

All analyses were conducted using R 4.0.1. A two-sided $p$ value of $<0.05$ was considered statistically significant.

\section{Results}

\section{Baseline characteristics of participants}

Table 1 presents baseline characteristics of the 266,280 participants with the mean age of 55.93 [standard deviation (SD): 8.08 ] years. The mean duration of follow-up was 10.64 (SD: 1.34) years. There were more females $(51.15 \%)$ than males and over 95\% participants were of White ethnicity. About $80 \%$ participants were classified as having moderate to vigorous PA levels. The mean annual average $\mathrm{PM}_{2.5}$ concentration in 2010 was higher in COPD incident cases [10.08 (SD 1.08) $\left.\mu \mathrm{g} / \mathrm{m}^{3}\right]$ than in non-cases [9.93 $\left.(\mathrm{SD} 1.04) \mu \mathrm{g} / \mathrm{m}^{3}\right]$. Detailed baseline information of the subsample with objectively measured PA was presented in Additional file 1: Table S2. The distribution of baseline characteristics was generally similar between the whole study population and the subsample.

\section{Association between self-reported PA and COPD incidence}

Table 2 shows the associations between self-reported PA and incident COPD. Higher PA level was associated with a lower incidence of COPD. The associations were robust to adjustment for various covariates, including $\mathrm{PM}_{2.5}$. In Model 4, compared with low PA level, the HR (95\% CI) of developing COPD was 0.769 (95\% CI: 0.720, 0.820) and 0.726 (95\% CI: $0.679,0.776)$ for moderate and high level of PA, respectively. In subgroup analysis, the negative association of self-reported PA and COPD incidence remained stable in participants exposed to different $\mathrm{PM}_{2.5}$ levels (Table 3).

\section{Association between chronic $\mathrm{PM}_{2.5}$ exposure and COPD incidence}

In contrast to $\mathrm{PA}$, positive associations of $\mathrm{PM}_{2.5}$ with COPD incidence were observed, and the associations remained unchanged after adjustment for PA and other covariates (Table 2). An IQR increment in $\mathrm{PM}_{2.5}$ was associated with a $6.5 \%(95 \%$ CI: $3.2 \%, 9.9 \%)$ increase in COPD 
Table 1 Baseline characteristics of participants in the UK Biobank Cohort

\begin{tabular}{|c|c|c|c|c|}
\hline Level $^{a}$ & Overall & Non-COPD case & COPD case & $P$ \\
\hline Total number & 266,280 & 259,846 & 6434 & - \\
\hline Follow-up duration (year) & $10.64(1.34)$ & $10.65(1.33)$ & $10.36(1.78)$ & $<0.001$ \\
\hline Age (year) & $55.93(8.08)$ & $55.81(8.08)$ & $61.16(6.50)$ & $<0.001$ \\
\hline \multicolumn{5}{|l|}{ Sex } \\
\hline Female & $136,199(51.15)$ & $133,445(51.36)$ & $2754(42.80)$ & \multirow[t]{2}{*}{$<0.001$} \\
\hline Male & $130,081(48.85)$ & $126,401(48.64)$ & $3680(57.20)$ & \\
\hline \multicolumn{5}{|l|}{ Ethnicity } \\
\hline Nonwhite & $11,852(4.45)$ & $11,682(4.50)$ & $170(2.64)$ & \multirow[t]{2}{*}{$<0.001$} \\
\hline White & $254,428(95.55)$ & $248,164(95.50)$ & $6264(97.36)$ & \\
\hline \multicolumn{5}{|l|}{ Household income $(£)$} \\
\hline Less than 18,000 & $49,790(18.70)$ & $47,325(18.21)$ & $2465(38.31)$ & \multirow[t]{5}{*}{$<0.001$} \\
\hline 18,000 to 30,999 & $65,324(24.53)$ & 63,385 (24.39) & $1939(30.14)$ & \\
\hline 31,000 to 51,999 & $72,779(27.33)$ & $71,571(27.54)$ & $1208(18.78)$ & \\
\hline 52,000 to 100,000 & $61,171(22.97)$ & $60,482(23.28)$ & $689(10.71)$ & \\
\hline Greater than 100,000 & $17,216(6.47)$ & $17,083(6.57)$ & $133(2.07)$ & \\
\hline \multicolumn{5}{|l|}{ Smoking status } \\
\hline Never & $157,380(59.10)$ & $155,431(59.82)$ & $1949(30.29)$ & \multirow[t]{3}{*}{$<0.001$} \\
\hline Previous & $100,909(37.90)$ & $96,756(37.24)$ & $4153(64.55)$ & \\
\hline Current & $7991(3.00)$ & $7659(2.95)$ & $332(5.16)$ & \\
\hline \multicolumn{5}{|l|}{ BMI level } \\
\hline Normal & $87,535(32.87)$ & $86,085(33.13)$ & $1450(22.54)$ & \multirow[t]{4}{*}{$<0.001$} \\
\hline Underweight & $1046(0.39)$ & $1015(0.39)$ & $31(0.48)$ & \\
\hline Overweight & $115,818(43.49)$ & $113,205(43.57)$ & $2613(40.61)$ & \\
\hline Obese & $61,881(23.24)$ & $59,541(22.91)$ & $2340(36.37)$ & \\
\hline \multicolumn{5}{|l|}{ Intake alcohol } \\
\hline Never & $17,322(6.51)$ & $16,753(6.45)$ & $569(8.84)$ & \multirow[t]{4}{*}{$<0.001$} \\
\hline Occasional & $54,536(20.48)$ & $53,073(20.42)$ & $1463(22.74)$ & \\
\hline Moderate & $136,262(51.17)$ & $133,417(51.34)$ & $2845(44.22)$ & \\
\hline Heavy & $58,160(21.84)$ & $56,603(21.78)$ & $1557(24.20)$ & \\
\hline PA MET (minute) & $2618.74(2640.64)$ & $2619.42(2635.74)$ & $2591.50(2831.24)$ & 0.40 \\
\hline \multicolumn{5}{|l|}{ Self-reported PA level } \\
\hline Low & $49,102(18.44)$ & $47,607(18.32)$ & $1495(23.24)$ & \multirow[t]{3}{*}{$<0.001$} \\
\hline Moderate & $109,332(41.06)$ & $106,802(41.10)$ & $2530(39.32)$ & \\
\hline High & $107,846(40.50)$ & $105,437(40.58)$ & $2409(37.44)$ & \\
\hline \multicolumn{5}{|l|}{ Education attainment } \\
\hline Any school degree & $103,206(38.76)$ & $101,080(38.90)$ & $2126(33.04)$ & \multirow[t]{4}{*}{$<0.001$} \\
\hline College education & $102,387(38.45)$ & $100,944(38.85)$ & $1443(22.43)$ & \\
\hline Vocational qualifications & $16,690(6.27)$ & $16,082(6.19)$ & $608(9.45)$ & \\
\hline Other & $43,997(16.52)$ & $41,740(16.06)$ & $2257(35.08)$ & \\
\hline \multicolumn{5}{|l|}{ Fruit and vegetable intake } \\
\hline Low & $71,338(26.79)$ & $69,503(26.75)$ & $1835(28.52)$ & \multirow[t]{3}{*}{0.007} \\
\hline Moderate & $138,311(51.94)$ & $135,051(51.97)$ & $3260(50.67)$ & \\
\hline High & $56,631(21.27)$ & $55,292(21.28)$ & $1339(20.81)$ & \\
\hline \multicolumn{5}{|l|}{ Employment status } \\
\hline Paid & $167,651(62.96)$ & $165,175(63.57)$ & $2476(38.48)$ & \multirow[t]{3}{*}{$<0.001$} \\
\hline Retired & $80,759(30.33)$ & $77,426(29.80)$ & $3333(51.80)$ & \\
\hline Unpaid & $17,870(6.71)$ & $17,245(6.64)$ & $625(9.71)$ & \\
\hline Townsend deprivation index & $-1.63(2.86)$ & $-1.65(2.85)$ & $-0.92(3.19)$ & $<0.001$ \\
\hline $\mathrm{PM}_{2.5}\left(\mu \mathrm{g} / \mathrm{m}^{3}\right)$ & $9.94(1.04)$ & $9.93(1.04)$ & $10.08(1.08)$ & $<0.001$ \\
\hline
\end{tabular}


Table 1 (continued)

Abbreviations: COPD chronic obstructive pulmonary diseases, $B M I$ body mass index, $P A$ physical activity, $P M_{2.5}$ particulate matter with an aerodynamic diameter $<2.5$ $\mu \mathrm{m}, M E T$ metabolic equivalents

${ }^{a}$ The statistics are shown as mean [standard deviation (SD)] for continuous variables and number (\%) for categorical variables

Table 2 Associations of habitual physical activity (PA) and long-term PM 2.5 exposure and COPD incidence $(n=6381)$ in UK Biobank Cohort

\begin{tabular}{|c|c|c|c|c|c|}
\hline Levels $^{a}$ & & Model I ${ }^{b}$ & Model $2^{b}$ & Model $3^{b}$ & Model $4^{b}$ \\
\hline Self-reported PA & \multicolumn{5}{|l|}{ Case } \\
\hline Low & 1495 & 1.000 & 1.000 & 1.000 & 1.000 \\
\hline Moderate & 2530 & $0.758(0.711,0.809)$ & $0.761(0.713,0.812)$ & $0.769(0.721,0.820)$ & $0.769(0.720,0.820)$ \\
\hline High & 2409 & $0.752(0.705,0.803)$ & $0.713(0.668,0.761)$ & $0.725(0.678,0.775)$ & $0.726(0.679,0.776)$ \\
\hline$P$ for trend & - & $<0.0001$ & $<0.0001$ & $<0.0001$ & $<0.0001$ \\
\hline Per 600 MET-min/week increment & 6434 & $0.990(0.985,0.996)$ & $0.986(0.980,0.991)$ & $0.987(0.981,0.993)$ & $0.987(0.982,0.993)$ \\
\hline \multicolumn{6}{|l|}{ Objectively measured PA } \\
\hline Low & 253 & 1.000 & 1.000 & 1.000 & 1.000 \\
\hline Moderate & 434 & $0.676(0.589,0.777)$ & $0.754(0.655,0.867)$ & $0.757(0.657,0.871)$ & $0.757(0.658,0.872)$ \\
\hline High & 403 & $0.542(0.462,0.636)$ & $0.637(0.540,0.752)$ & $0.635(0.538,0.750)$ & $0.635(0.538,0.750)$ \\
\hline$P$ for trend & - & $<0.0001$ & $<0.0001$ & $<0.0001$ & $<0.0001$ \\
\hline Per IQR increment & 1090 & $0.658(0.602,0.72)$ & $0.741(0.677,0.812)$ & $0.743(0.678,0.814)$ & $0.743(0.679,0.814)$ \\
\hline \multicolumn{6}{|l|}{$\mathrm{PM}_{2.5}$ levels } \\
\hline Low & 1959 & 1.000 & 1.000 & 1.000 & 1.000 \\
\hline Moderate & 2164 & $1.215(1.143,1.292)$ & $1.059(0.995,1.127)$ & $1.052(0.989,1.119)$ & $1.050(0.987,1.117)$ \\
\hline High & 2311 & $1.448(1.363,1.538)$ & $1.094(1.025,1.167)$ & $1.072(1.005,1.144)$ & $1.068(1.001,1.140)$ \\
\hline$P$ for trend & - & $<0.0001$ & 0.007 & 0.036 & 0.046 \\
\hline Per IQR increment $\left(1.27 \mu \mathrm{g} / \mathrm{m}^{3}\right)$ & 6434 & $1.243(1.209,1.278)$ & $1.080(1.046,1.115)$ & $1.067(1.034,1.101)$ & $1.065(1.032,1.099)$ \\
\hline
\end{tabular}

The bold type represents the statistically significant differences $(p<0.05)$

Abbreviations: $P M_{2.5}$ particulate matter with aerodynamic diameter $<2.5 \mu \mathrm{m}, \mathrm{HR}$ hazard ratio, $\mathrm{Cl}$ confidence interval, $P A$ physical activity, $C O P D$ chronic obstructive pulmonary disease

a : $\mathrm{PM}_{2.5}$ exposure levels (low, moderate, and high) were defined by $\mathrm{PM}_{2.5}$ tertiles $\left(<9.48 \mu \mathrm{g} / \mathrm{m}^{3}, 9.48\right.$ to $10.27 \mu \mathrm{g} / \mathrm{m}^{3}$, and $\left.\geq 10.27 \mu \mathrm{g} / \mathrm{m}^{3}\right)$, self-reported PA levels were defined according to a standard scoring criteria of International Physical Activity Questionnaire (IPAQ): low ( $<600 \mathrm{MET}$-min/week), moderate (600 to $3000 \mathrm{MET}$-min/ week), and high ( $\geq 3000 \mathrm{MET}-\mathrm{min} /$ week), and objectively measured PA levels were defined by objectively measured PA tertiles [<24.32 milli-gravity (mg), 24.32 to $30.70 \mathrm{mg}$, and $\geq 30.70 \mathrm{mg}]$

${ }^{\mathrm{b}}$ Model 1 was adjusted for age at enrolment, sex, and ethnicity. Model 2 was further adjusted for household income, employment status, education, and Townsend deprivation index. Model 3 was further adjusted for lifestyle factors (smoking status, alcohol intake frequency, fruit and vegetable intake), body mass index (BMI), and secondhand smoke exposure. And Model 4 was further mutually adjusted by PA (effect estimation of $\mathrm{PM}_{2.5}$ ) or $\mathrm{PM}_{2.5}$ (effect estimation of $\mathrm{PA}$ )

risk. In tertile-based analysis, compared with participants exposed to low level of $\mathrm{PM}_{2.5}$, the HR of having COPD was 1.050 (95\% CI: 0.987, 1.117) and 1.068 (95\% CI: 1.032, 1.140) for those exposed to moderate and high levels of $\mathrm{PM}_{2.5}$, respectively. The concentration-response curve for the association between $\mathrm{PM}_{2.5}$ and COPD is presented in Additional file 1: Figure S1. The positive associations remained in subgroups with different PA levels, though some were statistically non-significant (Table 4).

\section{Potential interaction between self-reported PA and $\mathrm{PM}_{2.5}$ on the COPD incidence}

Table 5 shows the combined health impact of selfreported PA level and chronic exposure to $\mathrm{PM}_{2.5}$ on
COPD incidence. Participants with higher PA or lower $\mathrm{PM}_{2.5}$ exposure generally had lower risk of COPD. Using the participants with low level of PA and high $\mathrm{PM}_{2.5}$ exposure as the reference, those with high PA level and low $\mathrm{PM}_{2.5}$ exposure had the lowest risk of developing COPD (HR: 0.681, 95\% CI: 0.607, 0.764).

Results of interaction between self-reported PA and $\mathrm{PM}_{2.5}$ on the incidence of COPD are presented in Additional file 1: Table S3, Table S4. On additive scale, little evidence of interaction was found (Additional file 1: Table S3). Similar results were also observed on multiplicative scale, with all $p$ values of the interaction term > 0.05 (Additional file 1: Table S4). 
Table 3 Associations between habitual PA levels and COPD incidence when exposed to different PM 2.5 levels

\begin{tabular}{|c|c|c|c|c|c|c|c|}
\hline \multirow[t]{2}{*}{ PA levels ${ }^{a}$} & \multirow[b]{2}{*}{ Case } & \multicolumn{2}{|l|}{ Low-level PM 2.5} & \multicolumn{2}{|l|}{ Moderate-level PM P.5 $_{2.5}$} & \multicolumn{2}{|l|}{ High-level PM 2.5} \\
\hline & & $\mathrm{HR}(95 \% \mathrm{Cl})^{b}$ & $P$ & $\mathrm{HR}(95 \% \mathrm{Cl})^{b}$ & $P$ & $\mathrm{HR}(95 \% \mathrm{Cl})^{\mathrm{b}}$ & $P$ \\
\hline \multicolumn{8}{|c|}{ Self-reported PA } \\
\hline Low & 1495 & 1.000 & - & 1.000 & - & 1.000 & - \\
\hline Moderate & 2530 & $0.786(0.698,0.884)$ & $<0.0001$ & $0.727(0.65,0.812)$ & $<0.0001$ & $0.792(0.711,0.882)$ & $<0.0001$ \\
\hline High & 2409 & $0.714(0.633,0.806)$ & $<0.0001$ & $0.707(0.631,0.792)$ & $<0.0001$ & $0.751(0.672,0.84)$ & $<0.0001$ \\
\hline Trend test & - & & $<0.0001$ & & $<0.0001$ & & $<0.0001$ \\
\hline \multicolumn{8}{|c|}{ Objectively measured PA } \\
\hline Low & 253 & 1.000 & - & 1.000 & - & 1.000 & - \\
\hline Moderate & 434 & $0.835(0.651,1.072)$ & 0.157 & $0.696(0.549,0.883)$ & 0.003 & $0.752(0.587,0.962)$ & 0.024 \\
\hline High & 403 & $0.656(0.489,0.881)$ & 0.005 & $0.571(0.429,0.76)$ & $<0.0001$ & $0.696(0.523,0.927)$ & 0.013 \\
\hline Trend test & - & & 0.005 & & 0.007 & & 0.001 \\
\hline
\end{tabular}

The bold type represents the statistically significant differences $(p<0.05)$

Abbreviations: $P M_{2.5}$ particulate matter with aerodynamic diameter $<2.5 \mu \mathrm{m}, H R$ hazard ratio, $C l$ confidence interval, $P A$ physical activity, $C O P D$ chronic obstructive pulmonary diseases

a Self-reported PA levels were defined according to a standard scoring criteria of International Physical Activity Questionnaire (IPAQ): low ( $<600 \mathrm{MET}$-min/week), moderate (600 to $3000 \mathrm{MET}-\mathrm{min} /$ week), and high ( $\geq 3000 \mathrm{MET}-\mathrm{min} /$ week), and objectively measured PA levels were defined by objectively measured PA tertiles [ $<$ 24.32 milli-gravity $(\mathrm{mg}), 24.32$ to $30.70 \mathrm{mg}$, and $\geq 30.70 \mathrm{mg}]$

${ }^{\mathrm{b}}$ All results were calculated fully adjusted by covariates in Model 3: age at enrolment, sex, ethnicity, household income, employment status, education, Townsend deprivation index, smoking status, alcohol intake frequency, fruit and vegetable intake, body mass index (BMI) and secondhand smoke exposure

Table 4 Associations between $\mathrm{PM}_{2.5}$ levels and COPD incidence when exposed to different self-reported physical activity (PA) levels

\begin{tabular}{|c|c|c|c|c|c|c|c|}
\hline \multirow[t]{2}{*}{$\mathrm{PM}_{2.5}$ levels $^{\mathrm{a}}$} & \multirow[b]{2}{*}{ Case } & \multicolumn{2}{|l|}{ Low-level PA } & \multicolumn{2}{|l|}{ Moderate-level PA } & \multicolumn{2}{|l|}{ High-level PA } \\
\hline & & $\mathrm{HR}(95 \% \mathrm{Cl})^{\mathrm{b}}$ & $P$ & $\mathrm{HR}(95 \% \mathrm{Cl})^{\mathrm{b}}$ & $P$ & $\mathrm{HR}(95 \% \mathrm{Cl})^{\mathrm{b}}$ & $P$ \\
\hline Low & 1959 & 1.000 & - & 1.000 & - & 1.000 & - \\
\hline Moderate & 2164 & $1.071(0.942,1.218)$ & 0.296 & $1.004(0.909,1.109)$ & 0.940 & $1.084(0.980,1.199)$ & 0.117 \\
\hline High & 2311 & $1.053(0.920,1.206)$ & 0.450 & $1.048(0.945,1.163)$ & 0.370 & $1.099(0.989,1.222)$ & 0.081 \\
\hline Trend test & - & & 0.464 & & 0.364 & & 0.081 \\
\hline
\end{tabular}

Abbreviations: $\mathrm{HR}$ hazard ratio, $\mathrm{Cl}$ confidence interval, $P A$ physical activity, $C O P D$ chronic obstructive pulmonary disease, $P M_{2.5}$ particulate matter with aerodynamic diameter $<2.5 \mu \mathrm{m}$

a $\mathrm{PM}_{2.5}$ exposure levels (low, moderate, and high) were defined by $\mathrm{PM}_{2.5}$ tertiles $\left(<9.48 \mu \mathrm{g} / \mathrm{m}^{3}, 9.48\right.$ to $10.27 \mu \mathrm{g} / \mathrm{m}^{3}$, and $\left.\geq 10.27 \mu \mathrm{g} / \mathrm{m}^{3}\right)$, and self-reported PA levels were defined according to a standard scoring criteria of International Physical Activity Questionnaire (IPAQ): low ( $<600 \mathrm{MET}-\mathrm{min} /$ week), moderate (600 to $3000 \mathrm{MET}-$ $\mathrm{min} /$ week), and high ( $\geq 3000 \mathrm{MET}-\mathrm{min} /$ week)

b All results were calculated fully adjusted by covariates in Model 3: age at enrolment, sex, ethnicity, household income, employment status, education, Townsend deprivation index, smoking status, alcohol intake frequency, fruit and vegetable intake, body mass index (BMI), and secondhand smoke exposure

\section{Results of objectively measured PA and COPD incidence}

Overall, similar results were found in analyses among the subsample with objectively measured PA. Higher level of objectively measured PA was associated with lower risk of COPD incidence (Table 2), and the negative association remained across different $\mathrm{PM}_{2.5}$ levels (Table 3). Participants with high PA level and low $\mathrm{PM}_{2.5}$ exposure had the lowest risk of developing COPD (HR: 0.574, 95\% CI: $0.432,0.764$ ) with reference to those with low level of PA and high level of $\mathrm{PM}_{2.5}$ exposure (Table 5). No interaction between objectively measured PA and $\mathrm{PM}_{2.5}$ were found, either on additive or multiplicative scale (Additional file 1: Table S3, Table S4).

\section{Sensitivity analyses}

The results of sensitivity analyses are presented in Additional file 1: Table S3-S9. Additional adjustment for baseline lung function or inverse distance to main road did not alter the results materially, especially for PA (Additional file 1: Table S3-S6). The associations between PA, long-term $\mathrm{PM}_{2.5}$ exposure, and incident $\mathrm{COPD}$ also remained robust when treating all-cause death as a competing risk (Additional file 1: Table S7). Furthermore, after the imputation of baseline missing data, the effect estimates of the associations between PA and long-term $\mathrm{PM}_{2.5}$ exposure and COPD incidence were similar as well (Additional file 1: Table S8, Table S9). 
Table 5 Combined effects of habitual physical activity, long-term PM $_{2.5}$ exposure, and COPD incidence in the UK Biobank

\begin{tabular}{|c|c|c|c|c|c|c|}
\hline \multirow[t]{2}{*}{ PA levels ${ }^{a}$} & \multicolumn{3}{|c|}{$\mathrm{PM}_{2.5}$ levels (HR, 95\% Cl) ${ }^{\mathrm{a}, \mathrm{b}}$} & \multicolumn{2}{|l|}{ RERI $^{c}$} & \multirow{2}{*}{$\begin{array}{l}P \text { for } \\
\text { interaction } \\
d\end{array}$} \\
\hline & High & Moderate & Low & Moderate $\mathrm{PM}_{2.5}$ level & Low $\mathrm{PM}_{2.5}$ level & \\
\hline \multicolumn{4}{|l|}{ Self-reported PA } & & & \multirow[t]{4}{*}{0.805} \\
\hline Low & 1.000 & $1.026(0.909,1.159)$ & $0.955(0.840,1.085)$ & & & \\
\hline Moderate & $0.792(0.711,0.881)$ & $0.753(0.674,0.840)$ & $0.747(0.667,0.836)$ & $-0.01(-0.18,0.17)$ & $0.02(-0.14,0.19)$ & \\
\hline High & $0.745(0.668,0.831)$ & $0.736(0.658,0.822)$ & $0.681(0.607,0.764)$ & $0.01(-0.16,0.18)$ & $0.02(-0.15,0.19)$ & \\
\hline \multicolumn{4}{|l|}{$\begin{array}{l}\text { Objectively measured } \\
\text { PA }\end{array}$} & & & \multirow[t]{4}{*}{0.998} \\
\hline Low & 1.000 & $1.088(0.885,1.337)$ & $0.923(0.740,1.152)$ & & & \\
\hline Moderate & $0.755(0.592,0.963)$ & $0.790(0.620,1.006)$ & $0.735(0.573,0.943)$ & $-0.05(-0.35,0.25)$ & $0.06(-0.23,0.34)$ & \\
\hline High & $0.693(0.527,0.911)$ & $0.653(0.492,0.866)$ & $0.574(0.432,0.764)$ & $-0.13(-0.44,0.18)$ & $0.02(-0.14,0.19)$ & \\
\hline \multicolumn{7}{|c|}{$\begin{array}{l}\text { Abbreviations: } H R \text { hazard ratio, } C I \text { confidence interval, } R E R I \text { relative excess risk due to interaction, } P A \text { physical activity, } P M_{2.5} \text { particulate matter with aerodynamic } \\
\text { diameter }<2.5 \mu \mathrm{m}, C O P D \text { chronic obstructive pulmonary disease }\end{array}$} \\
\hline \multicolumn{7}{|c|}{$\begin{array}{l}\text { a : } \mathrm{PM}_{2.5} \text { exposure levels (low, moderate, and high) were defined by } \mathrm{PM} \mathrm{M}_{2.5} \text { tertiles }\left(<9.48 \mu \mathrm{g} / \mathrm{m}^{3}, 9.48 \text { to } 10.27 \mu \mathrm{g} / \mathrm{m}^{3}, \text { and } \geq 10.27 \mu \mathrm{g} / \mathrm{m}^{3}\right) \text {, self-reported PA levels were } \\
\text { defined according to a standard scoring criteria of International Physical Activity Questionnaire (IPAQ): low }(<600 \mathrm{MET}-\mathrm{min} / \text { week), moderate }(600 \text { to } 3000 \mathrm{MET}-\mathrm{min} / \\
\text { week), and high ( } \geq 3000 \mathrm{MET} \text {-min/week), and objectively measured PA levels were defined by objectively measured PA tertiles }(<24.32 \mathrm{milli} \text {-gravity (mg), } 24.32 \text { to } \\
30.70 \mathrm{mg} \text {, and } \geq 30.70 \mathrm{mg} \text { ) }\end{array}$} \\
\hline \multicolumn{7}{|c|}{$\begin{array}{l}{ }^{\mathrm{b}} \text { All results were calculated based on covariates in Model } 3 \text { : age at enrolment, sex, ethnicity, BMI, education, household income, employment status, smoking status, } \\
\text { alcohol drinking, fruit and vegetable intake, secondhand smoke exposure, and Townsend deprivation index }\end{array}$} \\
\hline \multicolumn{7}{|c|}{${ }^{\mathrm{c}}$ The estimates of RERI were calculated based on the reference group with high level of $\mathrm{PM}_{2.5}$ exposure and low level of PA } \\
\hline
\end{tabular}

\section{Discussion}

To the best of our knowledge, this is the largest prospective cohort study so far to investigate the independent and combined health effects of habitual PA (measured by both questionnaire and accelerometer) and long-term $\mathrm{PM}_{2.5}$ exposure on the COPD incidence in adults. Higher level of habitual PA was significantly associated with lower risk of COPD incidence, regardless of the level of $\mathrm{PM}_{2.5}$ concentration. By contrast, positive associations between long-term $\mathrm{PM}_{2.5}$ exposure and COPD were observed. Little evidence of interaction between habitual PA and $\mathrm{PM}_{2.5}$ exposure on COPD development was observed, either on additive or multiplicative scale.

$\mathrm{PA}$ is a well-recognized protective factor against COPD [43, 44]. Consistent with previous findings [31, 45], we observed that higher levels of PA, both self-reported and objectively measured, were associated with a lower risk of COPD incidence. Another analysis using the UK Biobank data also reported a negative association between selfreported PA and COPD hospital admission (HR: 0.70, 95\% CI: 0.66, 0.75) [6]. According to previous studies, regular PA could reduce the risk of many adverse health outcomes partly because of its anti-inflammatory effect on the inflammatory process $[46,47]$.

The adverse health effects of exposure to $\mathrm{PM}_{2.5}$ on the development of COPD have been well documented [48-50]. Similarly, we also observed significant associations between exposure to $\mathrm{PM}_{2.5}$ and increased risk of COPD. Consistent with our findings, Doiron et al. reported that higher concentrations of $\mathrm{PM}_{2.5}$ was associated with increased COPD prevalence (OR: 1.52, 95\% CI: $1.42,1.62$ ) based on per $5 \mu \mathrm{g} / \mathrm{m}^{3}$ increment [6]. However, inconsistent results still remain. For example, in a large multi-country study, the ESCAPE (European Studies on Chronic Air Pollution Effects) study, no significant associations of $\mathrm{PM}_{2.5}$ with longitudinal change in lung function was observed [51]. Difference in health indicator may explain the heterogeneity to some extent: we focused on the incidence of COPD, while Adam et al. concentrated on the change in lung function metrics. Apart from the heterogeneity in study period, population, and location, the reasons behind the inconsistent findings require further investigations.

In analysis of combined effects of habitual PA and $\mathrm{PM}_{2.5}$ on COPD incidence, the negative associations between PA and COPD remained stable regardless of $\mathrm{PM}_{2.5}$ levels, which was in line with Kubesch et al's findings. Kubesch et al. [52] reported that, even in an environment with higher air pollution, intermittent moderate PA had beneficial effects on pulmonary function in a healthy population. Consistently, two studies based on the Danish Diet, Cancer, and Health Cohort yielded similar findings [53, 54], as both of them reported that the benefits of PA against asthma/COPD hospitalization were not attenuated by air pollution. For other health outcomes, two recent studies $[11,55]$ also 
reported independent associations of PA and air pollution with mortality or life expectancy. However, some other studies reported significant, but controversial results of the interactions between PA and air pollution on respiratory diseases. For example, in a cohort study in Taiwan [10], negative interactions between habitual PA and long-term exposure to $\mathrm{PM}_{2.5}$ were observed, as the beneficial health effects of PA on lung function were reportedly decreased by ambient $\mathrm{PM}_{2.5}$ exposure. By contrast, Matt et al. [56] reported that a one unit [1\% heart rate max (HRmax)] increase of PA was observed to reduce the immediate negative effects of particulate matter $\left(\mathrm{PM}_{2.5}\right)$ upon peak expiratory flow $(0.02 \mathrm{~L} / \mathrm{min})$. Similarly, Toledo et al. [57] suggested that regular moderate-intensity aerobic physical training attenuated the development of pulmonary diseases induced by cigarette smoke exposure. It is difficult to directly compare our results with the previous studies since the study period, targeted population, health indicators, and PA measurement varied. Further studies are warranted to better characterize the potential interactions between PA and air pollution.

The mechanisms underlying the potential interaction between $\mathrm{PA}$ and $\mathrm{PM}_{2.5}$ are currently unclear. Previous studies suggested that exercise of moderate intensity may improve immune responses to lower chronic low-grade inflammation and improve a variety of immune markers in several disease conditions $[58,59]$. There was also evidence suggesting that exposure to higher levels of air pollution could lead to declines in immunity stability through oxidative stress and chronic inflammation response [60]. The benefits of habitual PA may not be counteracted by the short-term elevated $\mathrm{PM}_{2.5}$ exposure during exercise, especially in areas where $\mathrm{PM}_{2.5}$ level are low to moderate, such as the UK [61]. Furthermore, the additional inhaled air pollutants due to PA only constitute a small fraction of the total inhaled air pollutants [62], which may not induce serious impairment on the respiratory system.

\section{Strengths and limitations of the study}

Our study has several important strengths. First, we used a large cohort of 266,280 participants with extensive information on a wide range of potential confounders, which enabled us to investigate the associations more reliably. More importantly, in addition to self-reported PA, we did an additional analysis in a subsample of nearly 100,000 participants who wore Axivity AX3 triaxial accelerometers to measure their daily PA duration and volume [24], and similar results were found. Such objective method provided more accurate measurements, which is a main challenge in studies using self-reported PA [13].
Our study also has some limitations. First, we only used the annual average $\mathrm{PM}_{2.5}$ concentration in 2010 as a proxy for the long-term $\mathrm{PM}_{2.5}$ exposure, which may have led to exposure misclassification. However, many previous studies suggested that the spatial distribution of $\mathrm{PM}_{2.5}$ generally remains stable in the same region over a period as long as $>10$ years $[27,29,63]$. In the UK, the annual average concentration of $\mathrm{PM}_{2.5}$ between 2010 and 2019 were relatively stable according to the Department for Environment Food \& Rural Affairs of the UK (https://www.gov.uk/gover nment/statistics/emissions-of-air-pollutants/emissions-ofair-pollutants-in-the-uk-summary). Therefore, the annual concentration of $\mathrm{PM}_{2.5}$ in 2010 could serve as a surrogate measure of long-term exposure over the study period. Despite the issue mentioned above, another potential limitation of the study is the exclusion of a relatively large number of participants due to missing data for covariates, which generated a relatively healthier cohort [participants in the analytical cohort were more likely to be younger and physically active (Additional file 1: Table S10)]. However, the difference was not substantial, especially the difference in $\mathrm{PM}_{2.5}$ (as small as $0.13 \mu \mathrm{g} / \mathrm{m}^{3}$ ), and it should not seriously bias our results. Furthermore, as an alternative approach, we additionally conducted multiple imputations for those missing covariates and the associations of PA and long-term $\mathrm{PM}_{2.5}$ exposure with COPD incidence remained stable (Additional file 1: Table S8 and Table S9). In addition, compared with the general population, the COPD prevalence was lower in the UK Biobank cohort [the UK Biobank: $0.4 \%$ for men, and 0.4\% for women; the UK general population: $3 \%$ for men, and $2 \%$ for women among participants between 55 and 64 years], which implies the evidence of a healthy volunteer bias [64]. However, it has been suggested that this may not influence the valid estimates of associations [6] since sufficiently large numbers of individuals with different levels of exposures were investigated with high internal validity [64], but may only affect the extrapolation and underestimate the associations of $\mathrm{PM}_{2.5}$ and COPD incidence in a general population [6]. Last, our study is based on a European cohort with a lower $\mathrm{PM}_{2.5}$ concentration [65], findings of our study may therefore not be generalizable to populations in areas with relatively high $\mathrm{PM}_{2.5}$ concentrations.

\section{Conclusions}

In conclusion, based on a large cohort study, we found that long-term exposure to $\mathrm{PM}_{2.5}$ was associated with higher risk of COPD incidence. By contrast, both selfreported and objectively measured PA were associated with lower risk of COPD regardless of the levels of $\mathrm{PM}_{2.5}$, indicating independent effects of $\mathrm{PA}$ and $\mathrm{PM}_{2.5}$ on COPD incidence. 


\section{Supplementary Information}

The online version contains supplementary material available at https://doi. org/10.1186/s12916-022-02274-8.

Additional file 1: Supplementary methods of covariates and 4-model analysis protocol. Table S1. Correlation between exposure variables and covariates (Spearman correlation coefficients). Table S2. Baseline information of the subsample with objectively measured PA. Table S3. Relative excess risk due to interaction (RERI, 95\% Cl) of long-term $\mathrm{PM}_{25}$ exposure and habitual PA levels. Table S4. Combined effects of long-term PM $\mathrm{PM}_{2.5}$ exposure levels and physical activity (PA) levels on COPD incidence on multiplicative scales. Table S5. Associations of $\mathrm{PM}_{2.5}$ exposure and COPD incidence adjusted by baseline lung function, inverse distance to major road, and both. Table S6. Combined effects of long-term PM $\mathrm{P}_{25}$ exposure and self-reported physical activity (PA) on COPD incidence. Table S7. Associations between self-reported PA, long-term $\mathrm{PM}_{25}$ exposure and COPD incidence by treating all-cause death as a competing risk. Table S8. Associations between self-reported PA, long-term $\mathrm{PM}_{25}$ exposure and COPD incidence after conducting multiple imputations for missing covariates. Table S9. Interaction between PA and long-term PM ${ }_{25}$ exposure on both additive and multiplicative scales after conducting multiple imputations for missing covariates $(N=357,603)$. Table $\mathbf{S 1 0}$. Baseline characteristics of the excluded and included participants. Figure S1. Concentration-response relationship between long-term $\mathrm{PM}_{2.5}$ exposure and COPD incidence.

\section{Acknowledgements}

We wish to acknowledge the UK Biobank Resource under Application Number 69550 and also gratefully acknowledge many participants who provided medical data to the UK Biobank.

\section{Authors' contributions}

LC conceived the idea and contributed to the analysis, interpretation, and draft of the work. MC contributed to the analysis of the data for the work. HTL contributed to the acquisition of the data. XJW, FT, and YLW revised the manuscript. ZLZ contributed to the interpretation of the work and edition of the manuscript. HLL conceived the idea and contributed to the acquisition of the data, interpretation of the work, and edition of the manuscript. All authors read and approved the final manuscript.

\section{Funding}

This work was supported, in whole or in part, by the Bill \& Melinda Gates Foundation [Grant Number: INV-016826]. Under the grant conditions of the foundation, a Creative Commons Attribution 4.0 Generic License has already been assigned to the Author Accepted Manuscript version that might arise from this submission.

\section{Availability of data and materials}

The datasets generated and analyzed during the current study are available upon reasonable request to the Access Management System (AMS) through the UK Biobank website (https://www.ukbiobank.ac.uk/enable-your-research/ apply-for-access).

\section{Declarations}

\section{Ethics approval and consent to participate}

The project has approval from the North West Multi-centre Research Ethics Committee (MREC) (REC reference: 21/NW/0157), and informed written consent was obtained from each participant.

\section{Consent for publication}

Not applicable.

\section{Competing interests}

The authors declare that they have no competing interests.

\section{Author details}

'Department of Epidemiology, School of Public Health, Sun Yat-sen University, Guangzhou 510080, China. ${ }^{2}$ Department of Social Medicine and Health
Service Management, Shenzhen University General Hospital, Shenzhen 518055, China.

Received: 10 October 2021 Accepted: 24 January 2022

Published online: 28 February 2022

\section{References}

1. Zhao Q, Li S, Coelho M, Saldiva PHN, Xu R, Huxley RR, et al. Ambient heat and hospitalisation for COPD in Brazil: a nationwide case-crossover study. Thorax. 2019;74(11):1031-6 https://thorax.bmj.com/content/thoraxjnl/ 74/11/1031.full.pdf.

2. GBD Chronic Respiratory Disease Collaborators. Prevalence and attributable health burden of chronic respiratory diseases, 1990-2017: a systematic analysis for the Global Burden of Disease Study 2017. Lancet Respir Med. 2020;8(6):585-96 https://www.ncbi.n/m.nih.gov/pmc/artic les/PMC7284317/.

3. Kyu HH, Abate D, Abate KH, Abay SM, Abbafati C, Abbasi N, et al. Global, regional, and national disability-adjusted life-years (DALYs) for 359 diseases and injuries and healthy life expectancy (HALE) for 195 countries and territories, 1990-2017: a systematic analysis for the Global Burden of Disease Study 2017. Lancet. 2018;392(10159):1859-922 https://www. ncbi.nlm.nih.gov/pmc/articles/PMC6252083/pdf/emss-80527.pdf.

4. Burge AT, Cox NS, Abramson MJ, Holland AE. Interventions for promoting physical activity in people with chronic obstructive pulmonary disease (COPD). Cochrane Database Syst Rev. 2020;4:CD012626 https://www. ncbi.nlm.nih.gov/pubmed/32297320.

5. Hendryx M, Luo J, Chojenta C, Byles JE. Air pollution exposures from multiple point sources and risk of incident chronic obstructive pulmonary disease (COPD) and asthma. Environ Res. 2019;179(Pt A):108783 https:// www.sciencedirect.com/science/article/pii/S0013935119305808?via\% 3Dihub.

6. Doiron D, de Hoogh K, Probst-Hensch N, Fortier I, Cai Y, De Matteis S, et al. Air pollution, lung function and COPD: results from the population-based UK Biobank study. Eur Respir J. 2019;54(1):1802140. https://erj.ersjournals. com/content/erj/54/1/1802140.full.pdf.

7. Cao D, Li D, Wu Y, Qian ZM, Liu Y, Liu Q, et al. Ambient PM(2.5) exposure and hospital cost and length of hospital stay for respiratory diseases in 11 cities in Shanxi Province, China. Thorax. 2021. https://doi.org/10.1136/ thoraxjnl-2020-215838.

8. Meshe OF, Claydon LS, Bungay H, Andrew S. The relationship between physical activity and health status in patients with chronic obstructive pulmonary disease following pulmonary rehabilitation. Disabil Rehabil. 2017;39(8):746-56 https://www.tandfonline.com/doi/pdf/10.3109/09638 288.2016.1161842? needAccess=true.

9. Zhang Z, Hoek G, Chang LY, Chan TC, Guo C, Chuang YC, et al. Particulate matter air pollution, physical activity and systemic inflammation in Taiwanese adults. Int J Hyg Environ Health. 2018;221(1):41-7 https://www. ncbi.nlm.nih.gov/pubmed/29030094.

10. Guo C, Bo Y, Chan TC, Zhang Z, Lin C, Tam T, et al. Lao XQ: Does fine particulate matter (PM2.5) affect the benefits of habitual physical activity on lung function in adults: a longitudinal cohort study. BMC Med. 2020;18(1):134 https://www.ncbi.nlm.nih.gov/pubmed/32398155.

11. Tainio M, Jovanovic Andersen Z, Nieuwenhuijsen MJ, Hu L, de Nazelle A, An $\mathrm{R}$, et al. Air pollution, physical activity and health: a mapping review of the evidence. Environ Int. 2021;147:105954 https://www.ncbi.nlm.nih. gov/pubmed/33352412.

12. Shephard RJ. Limits to the measurement of habitual physical activity by questionnaires. Br J Sports Med. 2003;37(3):197-206 https://bjsm.bmj. com/content/37/3/197.long.

13. Füzéki E, Engeroff T, Banzer W. Health benefits of light-intensity physical activity: a systematic review of accelerometer data of the National Health and Nutrition Examination Survey (NHANES). Sports Med. 2017;47(9):1769-93 https://link.springer.com/article/10.1007\%2Fs40 279-017-0724-0.

14. Gao M, Jebb SA, Aveyard P, Ambrosini GL, Perez-Cornago A, Carter J, et al. Associations between dietary patterns and the incidence of total and fatal cardiovascular disease and all-cause mortality in 116,806 individuals from the UK Biobank: a prospective cohort study. BMC Med. 2021;19(1):83 https://www.ncbi.nlm.nih.gov/pubmed/33882922. 
15. Sudlow C, Gallacher J, Allen N, Beral V, Burton P, Danesh J, et al. UK Biobank: an open access resource for identifying the causes of a wide range of complex diseases of middle and old age. PLoS Med. 2015;12(3):e1001779 https://journals.plos.org/plosmedicine/article?id= 10.1371/journal.pmed.1001779.

16. Hajna S, White T, Panter J, Brage S, Wijndaele K, Woodcock J, et al. Driving status, travel modes and accelerometer-assessed physical activity in younger, middle-aged and older adults: a prospective study of 90810 UK Biobank participants. Int J Epidemiol. 2019;48(4):1175-86 https://www. ncbi.nlm.nih.gov/pmc/articles/PMC6693808/pdf/dyz065.pdf.

17. Ho FK, Gray SR, Welsh P, Petermann-Rocha F, Foster H, Waddell H, et al. Associations of fat and carbohydrate intake with cardiovascular disease and mortality: prospective cohort study of UK Biobank participants. BMJ. 2020;368:m688 https://www.ncbi.nlm.nih.gov/pubmed/32188587.

18. Tikkanen E, Gustafsson S, Ingelsson E. Associations of fitness, physical activity, strength, and genetic risk with cardiovascular disease: longitudinal analyses in the UK Biobank Study. Circulation. 2018;137(24):2583-91 https://www.ncbi.nlm.nih.gov/pubmed/29632216.

19. Craig CL, Marshall AL, Sjöström M, Bauman AE, Booth ML, Ainsworth BE, et al. International physical activity questionnaire: 12-country reliability and validity. Med Sci Sports Exerc. 2003;35(8):1381-95 https://journals. Iww.com/acsm-msse/Fulltext/2003/08000/International_Physical_Activ ity_Questionnaire_.20.aspx.

20. IPAQ Research Committee. Guidelines for Data Processing and Analysis of the International Physical Activity Questionnaire (IPAQ) - Short and Long Forms. 2005. Available from: https://www.physio-pedia.com/images/c/ c7/Quidelines for interpreting the IPAQ.pdf. Accessed Nov 2005.

21. Chudasama YV, Khunti KK, Zaccardi F, Rowlands AV, Yates T, Gillies CL, et al. Physical activity, multimorbidity, and life expectancy: a UK Biobank longitudinal study. BMC Med. 2019;17(1):108 https://www.ncbi.nlm.nih. gov/pmc/articles/PMC6560907/pdf/12916_2019_Article_1339.pdf.

22. Hamer M, Sharma N, Batty GD. Association of objectively measured physical activity with brain structure: UK Biobank study. J Intern Med. 2018;284(4):439-43 https://onlinelibrary.wiley.com/doi/pdfdirect/10. 1111/joim.12772?download=true

23. Doherty A, Jackson D, Hammerla N, Plotz T, Olivier P, Granat MH, et al. Large scale population assessment of physical activity using wrist worn accelerometers: the UK Biobank Study. PLoS One. 2017;12(2):e0169649 https://www.ncbi.nlm.nih.gov/pubmed/28146576.

24. Ramakrishnan R, Doherty A, Smith-Byrne K, Rahimi K, Bennett D, Woodward M, et al. Accelerometer measured physical activity and the incidence of cardiovascular disease: evidence from the UK Biobank cohort study. PLoS Med. 2021;18(1):e1003487 https://www.ncbi.nlm.nih. gov/pmc/articles/PMC7802951/pdf/pmed.1003487.pdf.

25. Doiron D, Hoogh K, Probst-Hensch N, Mbatchou S, Eeftens M, Cai $Y$, et al. Residential air pollution and associations with wheeze and shortness of breath in adults: a combined analysis of cross-sectional data from two large European cohorts. Environ Health Perspect. 2017;125(9):097025 https://www.ncbi.nlm.nih.gov/pmc/articles/PMC59 15193/pdf/EHP1353.pdf.

26. Eeftens M, Beelen R, de Hoogh K, Bellander T, Cesaroni G, Cirach M, et al. Development of land use regression models for PM(2.5), PM(2.5) absorbance, PM(10) and PM(coarse) in 20 European study areas; results of the ESCAPE project. Environ Sci Technol. 2012;46(20):11195-205.

27. Huang Y, Zhu M, Ji M, Fan J, Xie J, Wei X, et al. Air pollution, genetic factors, and the risk of lung cancer: a prospective study in the UK Biobank. Am J Respir Crit Care Med. 2021;204(7):817-25 https://www.atsjournals. org/doi/10.1164/rccm.202011-4063OC?url_ver=Z39.88-2003\&rfr_id=ori: rid:crossref.org\&rfr_dat=cr_pub\%20\%200pubmed.

28. Qiu H, Sun S, Tsang H, Wong CM, Lee RS, Schooling CM, et al. Fine particulate matter exposure and incidence of stroke: a cohort study in Hong Kong. Neurology. 2017;88(18):1709-17 https://n.neurology.org/content/ 88/18/1709.long.

29. Furlong MA, Klimentidis YC. Associations of air pollution with obesity and body fat percentage, and modification by polygenic risk score for BMI in the UK Biobank. Environ Res. 2020;185:109364 https://www.ncbi.nlm.nih. gov/pmc/articles/PMC7199644/pdf/nihms-1581385.pdf.

30. Karakatsani A, Analitis A, Perifanou D, Ayres JG, Harrison RM, Kotronarou $A$, et al. Particulate matter air pollution and respiratory symptoms in individuals having either asthma or chronic obstructive pulmonary disease: a European multicentre panel study. Environ Health. 2012;11:75
https://www.ncbi.nlm.nih.gov/pmc/articles/PMC3509003/pdf/1476069X-11-75.pdf.

31. Pearce M, Strain T, Kim Y, Sharp SJ, Westgate K, Wijndaele K, et al. Estimating physical activity from self-reported behaviours in large-scale population studies using network harmonisation: findings from UK Biobank and associations with disease outcomes. Int J Behav Nutr Phys Act. 2020;17(1):40 https://www.ncbi.nlm.nih.gov/pmc/articles/PMC7074990/ pdf/12966_2020_Article_937.pdf.

32. Niedzwiedz CL, O'Donnell CA, Jani BD, Demou E, Ho FK, Celis-Morales C, et al. Ethnic and socioeconomic differences in SARS-CoV-2 infection: prospective cohort study using UK Biobank. BMC Med. 2020;18(1):160 https://www.ncbi.nlm.nih.gov/pubmed/32466757.

33. Bhattacharya R, Zekavat SM, Uddin MM, Pirruccello J, Niroula A, Gibson C, et al. Association of Diet Quality With Prevalence of Clonal Hematopoiesis and Adverse Cardiovascular Events. JAMA Cardiol. 2021;6(9):1069-77. https://jamanetwork.com/journals/jamacardiology/fullarticle/2780386.

34. von Elm E, Altman DG, Egger M, Pocock SJ, Gøtzsche PC, Vandenbroucke JP. The Strengthening the Reporting of Observational Studies in Epidemiology (STROBE) Statement: guidelines for reporting observational studies. Int J Surg. 2014;12(12):1495-9 https://www.sciencedirect.com/science/ article/pii/S1743919114002131?via\%3Dihub.

35. Wen C, Hu H, Ou YN, Bi YL, Ma YH, Tan L, et al. Risk factors for subjective cognitive decline: the CABLE study. Transl Psychiatry. 2021;11(1):576 https://www.nature.com/articles/s41398-021-01711-1.pdf.

36. VanderWeele TJ, Knol MJ. A tutorial on interaction. Epidemiol Methods. 2014;3:1.

37. Chu H, Nie L, Cole SR. Estimating the relative excess risk due to interaction: a bayesian approach. Epidemiology. 2011;22(2):242-8 https://www. ncbi.nlm.nih.gov/pubmed/21228700.

38. Sun S, Cao W, Qiu H, Ran J, Lin H, Shen C, et al. Benefits of physical activity not affected by air pollution: a prospective cohort study. Int Epidemiol. 2020;49(1):142-52 https://academic.oup.com/ije/article/ 49/1/142/5559550.

39. Stiles VH, Metcalf BS, Knapp KM, Rowlands AV. A small amount of precisely measured high-intensity habitual physical activity predicts bone health in pre- and post-menopausal women in UK Biobank. Int J Epidemiol. 2017;46(6):1847-56 https://www.ncbi.nlm.nih.gov/pmc/artic les/PMC5837700/pdf/dyx080.pdf.

40. Kwon DS, Choi YJ, Kim TH, Byun MK, Cho JH, Kim HJ, et al. FEF(25-75\%) values in patients with normal lung function can predict the development of chronic obstructive pulmonary disease. Int J Chron Obstruct Pulmon Dis. 2020;15:2913-21.

41. Morici G, Cibella F, Cogo A, Palange P, Bonsignore MR. Respiratory effects of exposure to traffic-related air pollutants during exercise. Front Public Health. 2020;8:575137 https://www.ncbi.nlm.nih.gov/pmc/articles/ PMC7793908/pdf/fpubh-08-575137.pdf.

42. Honigberg MC, Zekavat SM, Aragam K, Finneran P, Klarin D, Bhatt DL, et al. Association of premature natural and surgical menopause with incident cardiovascular disease. Jama. 2019;322(24):2411-21 https://jamanetwork. com/journals/jama/articlepdf/2755841/jama_honigberg_2019_oi_ 190136.pdf.

43. Qin F, Yang Y, Wang ST, Dong YN, Xu MX, Wang ZW, et al. Exercise and air pollutants exposure: a systematic review and meta-analysis. Life Sci. 2019;218:153-64

44. Garcia Aymerich J. Physical activity and COPD development. Time to advocate. Thorax. 2019;74(9):831-2 https://www.ncbi.nlm.nih.gov/pub$\mathrm{med} / 31363022$

45. de Roos P, Lucas C, Strijbos JH, van Trijffel E. Effectiveness of a combined exercise training and home-based walking programme on physical activity compared with standard medical care in moderate COPD: a randomised controlled trial. Physiotherapy. 2018;104(1):116-21 https://www. sciencedirect.com/science/article/pii/S0031940617300603?via\%3Dihub.

46. Nimmo MA, Leggate M, Viana JL, King JA. The effect of physical activity on mediators of inflammation. Diabetes Obes Metab. 2013;15(Suppl 3):51-60 https://dom-pubs.onlinelibrary.wiley.com/doi/10.1111/dom.12156.

47. Fedewa MV, Hathaway ED, Ward-Ritacco CL. Effect of exercise training on $C$ reactive protein: a systematic review and meta-analysis of randomised and non-randomised controlled trials. Br J Sports Med. 2017;51(8):670-6 https://bjsm.bmj.com/content/51/8/670.long.

48. Lamichhane DK, Leem JH, Kim HC. Associations between Ambient Particulate Matter and Nitrogen Dioxide and Chronic Obstructive Pulmonary 
Diseases in Adults and Effect Modification by Demographic and Lifestyle Factors. Int J Environ Res Public Health. 2018;15(2):363. https://res.mdpi. com/d_attachment/ijerph/ijerph-15-00363/article_deploy/ijerph-1500363-v2.pdf.

49. Cole-Hunter T, de Nazelle A, Donaire-Gonzalez D, Kubesch N, Carrasco-Turigas G, Matt F, et al. Estimated effects of air pollution and space-time-activity on cardiopulmonary outcomes in healthy adults: a repeated measures study. Environ Int. 2018;111:247-59 https://spiral. imperial.ac.uk:8443/bitstream/10044/1/56552/8/ENVINT_2017_1466 rev_nomarkup.pdf.

50. Gu J, Shi Y, Zhu Y, Chen N, Wang H, Zhang Z, et al. Ambient air pollution and cause-specific risk of hospital admission in China: a nationwide timeseries study. PLoS Med. 2020;17(8):e1003188 https://journals.plos.org/ plosmedicine/article?id=10.1371/journal.pmed.1003188.

51. Adam M, Schikowski T, Carsin AE, Cai Y, Jacquemin B, Sanchez M, et al. Adult lung function and long-term air pollution exposure. ESCAPE: a multicentre cohort study and meta-analysis. Eur Respir J. 2015;45(1):38-50 https://www.ncbi.nlm.nih.gov/pubmed/25193994.

52. Kubesch NJ, de Nazelle A, Westerdahl D, Martinez D, Carrasco-Turigas $G$, Bouso $L$, et al. Respiratory and inflammatory responses to short-term exposure to traffic-related air pollution with and without moderate physical activity. Occup Environ Med. 2015;72(4):284-93 https://oem.bmj.com/ content/oemed/72/4/284.full.pdf.

53. Fisher JE, Loft S, Ulrik CS, Raaschou-Nielsen O, Hertel O, Tjønneland A, et al. Physical activity, air pollution, and the risk of asthma and chronic obstructive pulmonary disease. Am J Respir Crit Care Med. 2016;194(7):855-65 https://www.atsjournals.org/doi/10.1164/rccm. 201510-20360C?url_ver=Z39.88-2003\&rfr_id=ori:rid:crossref.org\&rfr_ dat=cr_pub\%20\%200pubmed.

54. Andersen ZJ, de Nazelle A, Mendez MA, Garcia-Aymerich J, Hertel O, Tjønneland A, et al. A study of the combined effects of physical activity and air pollution on mortality in elderly urban residents: the Danish Diet, Cancer, and Health Cohort. Environ Health Perspect. 2015;123(6):557-63 https://spiral.imperial.ac.uk:8443/bitstream/10044/1/32740/7/ehp.14086 98.alt.pdf.

55. Cepeda M, Schoufour J, Freak-Poli R, Koolhaas CM, Dhana K, Bramer WM, et al. Levels of ambient air pollution according to mode of transport: a systematic review. Lancet Public Health. 2017;2(1):e23-34 https://www. sciencedirect.com/science/article/pii/S2468266716300214?via\%3Dihub.

56. Matt F, Cole-Hunter T, Donaire-Gonzalez D, Kubesch N, Martínez D, Carrasco-Turigas $\mathrm{G}$, et al. Acute respiratory response to traffic-related air pollution during physical activity performance. Environ Int. 2016:97:4555 https://www.sciencedirect.com/science/article/pii/S01604120163057 97?via\%3Dihub.

57. Toledo AC, Magalhaes RM, Hizume DC, Vieira RP, Biselli PJ, Moriya HT, et al. Aerobic exercise attenuates pulmonary injury induced by exposure to cigarette smoke. Eur Respir J. 2012;39(2):254-64 https://erj.ersjournals. com/content/erj/39/2/254.full.pdf.

58. Simpson RJ, Campbell JP, Gleeson M, Krüger K, Nieman DC, Pyne DB, et al. Can exercise affect immune function to increase susceptibility to infection? Exerc Immunol Rev. 2020;26:8-22 http://eir-isei.de/2020/eir-2020008-article.pdf.

59. Pedersen BK. Anti-inflammatory effects of exercise: role in diabetes and cardiovascular disease. Eur J Clin Invest. 2017;47(8):600-11 https://onlin elibrary.wiley.com/doi/10.1111/eci.12781.

60. Lin H, Guo Y, Ruan Z, Yang Y, Chen Y, Zheng Y, et al. Ambient PM(2.5) and $\mathrm{O}(3)$ and their combined effects on prevalence of presbyopia among the elderly: A cross-sectional study in six low- and middle-income countries. Sci Total Environ. 2019;655:168-73 https://www.sciencedirect.com/scien ce/article/pii/S0048969718345959?via\%3Dihub.

61. Mukherjee A, Agrawal M. A global perspective of fine particulate matter pollution and its health effects. Rev Environ Contam Toxicol. 2018;244:551 https://link.springer.com/content/pdf/10.1007\%2F398_2017_3.pdf.

62. Rojas-Rueda D, de Nazelle A, Tainio M, Nieuwenhuijsen MJ. The health risks and benefits of cycling in urban environments compared with car use: health impact assessment study. BMJ. 2011;343:d4521 https://www. bmj.com/content/bmj/343/bmj.d4521.full.pdf.

63. Li X, Wang M, Song $Y, M a ~ H, Z$, hou T, Liang Z, et al. Obesity and the relation between joint exposure to ambient air pollutants and incident type 2 diabetes: a cohort study in UK Biobank. PLoS Med. 2021;18(8):e1003767 https://journals.plos.org/plosmedicine/article?id=10.1371/journal.pmed. 1003767.

64. Fry A, Littlejohns TJ, Sudlow C, Doherty N, Adamska L, Sprosen T, et al. Comparison of sociodemographic and health-related characteristics of UK Biobank participants with those of the general population. Am J Epidemiol. 2017;186(9):1026-34 https://www.ncbi.nlm.nih.gov/pmc/artic les/PMC5860371/pdf/kwx246.pdf

65. Zhang S, Li G, Tian L, Guo Q, Pan X. Short-term exposure to air pollution and morbidity of COPD and asthma in East Asian area: a systematic review and meta-analysis. Environ Res. 2016;148:15-23 https://www.scien cedirect.com/science/article/pii/S0013935116300883?via\%3Dihub.

\section{Publisher's Note}

Springer Nature remains neutral with regard to jurisdictional claims in published maps and institutional affiliations.
Ready to submit your research? Choose BMC and benefit from:

- fast, convenient online submission

- thorough peer review by experienced researchers in your field

- rapid publication on acceptance

- support for research data, including large and complex data types

- gold Open Access which fosters wider collaboration and increased citations

- maximum visibility for your research: over $100 \mathrm{M}$ website views per year

At BMC, research is always in progress.

Learn more biomedcentral.com/submissions 